\title{
Antimicrobial screening of Plectranthus madagascariensis and P. neochilus extracts
}

\author{
Pesquisa da actividade antimicrobiana de extratos de Plectranthus madagascariensis e P. \\ neochilus
}

\author{
Margarida Pereira, Diogo Matias, Filipe Pereira, Catarina P. Reis, M. Fátima Simões and Patrícia Rijo \\ CBIOS, Universidade Lusófona de Humanidades e Tecnologias, Campo Grande, 376, 1749-024 Lisboa, Portugal \\ Email: patricia.rijo@ulusofona.pt
}

\begin{abstract}
Natural products are widely used as traditional medicines and are a common source of bioactive molecules for the treatment of bacterial infections. In particular, some plants of the genus Plectranthus (Lamiaceae) have demonstrated several applications, including the treatment of various infections. In this work, aqueous, acetonic and methanolic extracts of P. madagascariensis and P. neochilus were prepared using several extraction methods (infusion, decoction, maceration, microwave- and ultrasoundassisted and supercritical fluids). All extracts were screened for their antimicrobial activity against Gram positive bacteria (Enterococcus faecalis, Staphylococcus aureus, Bacillus subtilis and Mycobacterium smegmatis) and Gram negative bacteria (Pseudomonas aeruginosa, Klebsiella pneumoniae and Escherichia coli) and two yeast (Candida albicans and Saccharomyces cerevisiae) strains. The P. madagascariensis acetonic extracts obtained using ultrasound and maceration methods and P. neochilus acetonic extract obtained by ultrasound technique showed activity against the five tested Gram positive bacteria (5-24 mm of inhibition zone using the well diffusion test). The antimicrobial activity was further evaluated by the microdilution method (MIC values were between $250-0.49 \mu \mathrm{g} / \mathrm{mL}$ ) and the bioautography assay against $\mathrm{S}$. aureus.

The P. madagascariensis ultrasound acetonic extract was the most active extract against all the tested Gram positive bacteria (MIC values ranged between $31.25-0.49 \mu \mathrm{g} / \mathrm{mL}$ ). It was also active against resistant MRSA and VRE strains (MIC values ranged between $31.25-0.98 \mu \mathrm{g} / \mathrm{mL}$ ). The $\mathrm{S}$. aureus bioautography assay showed that the more polar compounds were the responsible for the antimicrobial activity.
\end{abstract}

Keywords: Antimicrobial activity; natural products; Plectranthus spp; extraction methods.

\section{Resumo}

Os produtos naturais são amplamente utilizados como remédios tradicionais e são uma fonte comum de moléculas bioativas para o tratamento de infeções bacterianas. Em particular, algumas plantas do género Plectranthus (Lamiaceae) demonstraram diversas aplicações, incluindo no tratamento de várias infeções. Neste trabalho, foram preparados extratos aquosos, acetónicos e metanólicos de P. madagascariensis e P. neochilus utilizando vários métodos de extração (infusão, decocção, maceração, extração assistida por micro-ondas e por ultrassom e com fluidos no estado supercrítico). Todos os extratos foram testados quanto à atividade antimicrobiana face a bactérias de Gram-positivo (Enterococcus faecalis, Staphylococcus aureus, Staphylococcus epidermidis Bacillus subtilis e Mycobacterium smegmatis) e de Gram-negativo (Pseudomonas aeruginosa, Klebsiella pneumoniae e Escherichia coli) e duas estirpes de leveduras (Candida albicans e Saccharomyces cerevisiae). O extrato acetónico de P. madagascariensis obtido pelas técnicas de ultrassom e maceração e o extrato acetónico de P. neochilus obtido pela técnica de ultrassom demonstraram atividade contra as cinco bactérias Gram-positivas testadas (5-24 mm de zona de inibição no teste de difusão). A atividade antimicrobiana foi ainda avaliada pelo método da microdiluição (valores de CMI entre 250-0,49 $\mu \mathrm{g} / \mathrm{mL}$ ) e pelo ensaio de bioautografia usando o S. aureus. O extrato acetónico de P. madagascariensis foi o mais ativo contra todas as bactérias de Gram positivo testadas (valores de CMI entre 31,25-0,49 $\mu \mathrm{g} / \mathrm{ml}$ ). Foi também ativo em bactérias resistentes a antibióticos: MRSA e VRE (valores de CMI entre 31,25-0,98 $\mu \mathrm{g} / \mathrm{mL}$ ). Os ensaios de bioautografia com S. aureus demonstraram que os compostos mais polares são os responsáveis pela sua atividade antimicrobiana.

Palavras-chave: Actividade antimicrobiana; Produtos naturais; Plectranthus spp; Métodos de extração. 


\section{Introduction}

The medicinal plants, which are the basis of the traditional medicine, have been intensely studied in the recent decades. Many of those works were dedicated to the antimicrobial action of the plants and, lately, to the discovery of potential and new antimicrobial agents ${ }^{1,2}$.

The antimicrobials of natural origin usually present complex chemical structures which may be important for specific interactions and recognition of potential macromolecular targets in the pathogenic microorganisms. These active natural products are, generally, plant secondary metabolites biosynthesized to fight and prevent the proliferation of plant pathogenic microorganisms ${ }^{1}$.

In the developing countries it is estimated that about $80 \%$ of the population depends on traditional medicine for the primary health care ${ }^{2-3}$. Thus, there is an increased interest for screening the medicinal plants to identify the bioactive components of potential therapeutic value for the discovery and development of new drugs ${ }^{3}$.

The family of Lamiaceae contains various genera, such as Mentha (mint), Salvia (sage) and Ocimum (basil), which have a great commercial importance mainly due to their aromatic characteristics. Plectranthus is another important genus of this plant family that is widely found in Africa, Asia and Australia. It comprises about 300 species, 62 of which are reported as having food usage and therapeutic use, namely as antimicrobial agents against mycobacteria, bacteria, viruses, protozoa and fungi ${ }^{2,4-5}$.

The phytochemical studies on some species of Plectranthus revealed the presence of a large number of diterpenes and triterpenes ${ }^{2}$.

Diterpenes are natural compounds with a hydrocarbon backbone with twenty carbon atoms, corresponding to four isoprene units (a chemical structure of five carbon atoms). They may have an acyclic structure but most of them are cyclic compounds. Diterpenes are the second major group of terpenes resulting from the plant metabolism. They have several significant roles in the growth and development of the plant and are also important in the resistance to environmental stress ${ }^{2}$.

More than two thousands of diterpenoid compounds are known with diverse structures. They show a wide array of pharmacological activities including bronchodilator, anti-hypertensive, anti-inflammatory and platelet aggregation inhibitory properties. These properties are likely related to the hydrocarbon backbone cyclization (bicyclic, tricyclic, tetracyclic or macrocyclic) combined with a wide range of oxidized functional groups (e.g., hydroxyl and carbonyl groups) ${ }^{2}$.

Several phytochemical studies have reported the isola-

\section{Introdução}

As plantas medicinais que constituem a base da medicina tradicional têm sido, nas últimas décadas, objeto de intenso estudo farmacológico. Muitos destes trabalhos dedicaram-se ao estudo da ação antimicrobiana das plantas com o objetivo de contribuir para a descoberta de novos antimicrobianos. ${ }^{[1][2]}$

Os metabolitos antimicrobianos de origem natural apresentam geralmente estruturas químicas complexas eventualmente importantes para as interações específicas e reconhecimento dos alvos macromoleculares das bactérias patogénicas. Estes produtos naturais correspondem, geralmente, a metabolitos secundários biossintetizados para combater e prevenir a proliferação de microrganismos patogénicos para as plantas. ${ }^{[1]}$

Nos países em desenvolvimento estima-se que cerca de $80 \%$ da população dependa da medicina tradicional para os cuidados de saúde primários. ${ }^{[2][3]}$ Por conseguinte, a pesquisa de compostos bioactivos, com eventual interesse terapêutico, a partir das plantas medicinais é do maior interesse para a descoberta e o desenvolvimento de novos fármacos. ${ }^{[3]}$

A família de plantas Lamiaceae é constituída por vários géneros, tais como a Mentha (menta), Salvia (sálvia) e Ocimum (manjericão), que possuem uma grande importância comercial especialmente devido às suas características aromáticas. Outro género, também muito importante desta família de plantas, é o género Plectranthus que se encontra amplamente distribuído por África, Ásia e Austrália. Este género inclui mais de 300 espécies estando 62 destas descritas como tendo uso alimentar e uso terapêutico, nomeadamente, como agentes anti-infecciosos para combater micobactérias, bactérias, vírus, protozoários e fungos. ${ }^{[2][4-5]}$

Os estudos fitoquímicos em algumas espécies de Plectranthus revelaram a presença de uma grande variedade de componentes incluindo diterpenos e triterpenos. ${ }^{[2]}$

Os diterpenos são compostos naturais com um esqueleto hidrocarbonado constituído por vinte átomos de carbono, o que corresponde a quatro unidades de isopreno (uma estrutura química de cinco carbonos). Podem possuir uma estrutura acíclica, mas na maior parte são compostos cíclicos e são o segundo maior grupo de terpenos resultante do metabolismo das plantas. Têm diversos papéis relevantes, nomeadamente, no crescimento e desenvolvimento da planta e são importantes na resistência ao stress ambiental..$^{[2]}$

São conhecidos mais de dois mil compostos diterpénicos com estruturas distintas, apresentando uma ampla gama de propriedades farmacológicas, incluindo a broncodilatadora, anti-hipertensora, anti-inflamatória e 
tion and characterization of diterpenes, mostly abietanes with a royleanone substructure, showing antimicrobial activities. However, only a few studies have related the diterpene activity with the traditional use of the plant species $^{6-7}$.

Some species of the Plectranthus genus have ethnopharmacological applications, with antimicrobial activity often cited ${ }^{8}$. Indeed, it is known that both the metabolites and extracts of various Plectranthus spp. have exhibited activity against Gram-positive and Gramnegative bacteria and yeasts ${ }^{3-4,9}$.

This work aimed to study the microbial properties of Plectranthus madagascariensis and Plectranthus neochilus. Thus, the different antimicrobial activities of the extracts obtained by different solvents (water, acetone or methanol) and using different extraction methods (infusion, decoction, maceration, microwave or ultrasound) were examined. These activities were evaluates using the well diffusion assay in solid medium, the bioautography method and through the determination of the minimum inhibitory concentration (MIC) in liquid medium.

\section{Material and Methods}

\section{$\underline{\text { Plant material }}$}

Plectranthus madagascarensis and Plectranthus neochilus were provided by the Faculty of Pharmacy of the University of Lisbon.

\section{Extraction methodologies of the plant material}

The aqueous plant extracts were prepared using $10 \mathrm{~g}$ of powdered plant material dissolved in $100 \mathrm{~mL}$ of distilled water, subsequently filtered using Whatman paper n. $^{\circ} 5$ (Whatman, Inc., Clifton, NJ.). These aqueous extracts were obtained using three extraction methods: infusion, decoction and microwave-assisted.

The acetone and methanol extracts were prepared using inibidora da agregação plaquetária. A multiplicidade de ações farmacológicas dos diterpenos está relacionada com a diversidade de ciclização do esqueleto (bicíclico, tricíclico, tetracíclico ou macrocíclico) aliada a uma vasta gama de grupos funcionais com oxigénio (por exemplo grupos hidroxilo e grupos carbonilo)..$^{[2]}$

A maioria dos estudos fitoquímicos realizados teve como finalidade o isolamento e a caracterização destes compostos. Embora alguns diterpenos tenham demonstrado uma potente actividade antimicrobiana, como por exemplo os abietanos com estrutura de roileanona, poucos foram os estudos efectuados que relacionassem esta característica com o uso tradicional de certas espécies

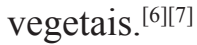

Algumas espécies de Plectranthus com aplicações etnofarmacológicas são usadas pela sua acção antimicrobiana. Muitas atividades biológicas têm sido atribuídas a plantas deste género e a sua atividade antimicrobiana é frequentemente citada ${ }^{[8]}$. Com efeito, sabe-se que tanto os extratos como os metabolitos de vários Plectranthus spp. apresentam frequentemente actividades contra bactérias de Gram-positivo, Gram-negativo e leveduras. ${ }^{[3][4][9] .}$

Este trabalho teve como objectivo estudar as características antimicrobianas das plantas Plectranthus madagascariensis e Plectranthus neochilus. Assim, pesquisaram-se as diferenças na actividade biológica resultantes da utilização de diferentes solventes de extracção (água, acetona e metanol) e diferentes métodos de extracção (infusão, decocção, maceração, microondas e ultrassons). A actividade antibacteriana foi determinada através do ensaio de difusão em poços através de meio sólido, do método de bioautografia e do método da determinação da concentração inibitória mínima (MIC) em meio líquido.

\section{Materiais e Métodos}

\section{Material vegetal}

Plectranthus madagascarensis e Plectranthus neochilus, foram fornecidos pela Faculdade de Farmácia da Universidade de Lisboa.

\section{Metodologias de extracção do material vegetal}

Os extratos vegetais aquosos foram preparados usando $10 \mathrm{~g}$ de material vegetal em pó colocado em $100 \mathrm{~mL}$ de água destilada, sendo posteriormente filtrado utilizando um papel de filtro Whatman n. ${ }^{\circ} 5$ (Whatman, Inc., Clifton, NJ, EUA). Estes extratos foram obtidos através de três métodos de extracção distintos: infusão, decocção e assistidos por micro-ondas. 
$10 \mathrm{~g}$ of powdered plant material dissolved in $200 \mathrm{~mL}$ of the respective solvents. These extracts were obtained using maceration extraction method and sonication.

In the decoction method the extracts were prepared boiling the plant material in distilled water for $10 \mathrm{~min}$ utes. In the infusion method, the extracts were prepared by addition of boiling distilled water to the plant for 10 minutes. The extracts prepared by the microwaveassisted method were obtained mixing the plant material with distilled water into a microwave oven for 2 minutes at a continuous irradiation at $2.45 \mathrm{GHz}$. In the maceration method, the plant material and the solvent were left under stirring at room temperature. After 24 hours, the plant material was filtered into a round bottom flask and then evaporated (vacuum at $40{ }^{\circ} \mathrm{C}$ ). In the ultrasonic method, the Erlenmeyer with the plant material and the solvent was placed in an ultrasonic bath at room temperature for 2 hours.

\section{Microorganisms}

The microorganisms used throughout this study were antibiotic sensitive (Bacillus subtilis ATCC 6633, Enterococcus faecalis ATCC 29212, Escherichia coli ATCC 25922, Pseudomonas aeruginosa ATCC 27853, Staphylococcus aureus ATCC 25923, Candida albicans ATCC 10231; Mycobacterium smegmatis ATCC 607; Klebsiella pneumoniae ATCC 9997; Saccharomyces cerevivisiae ATCC 9763 and Staphylococcus epidermidis ATCC 12228) and antibiotic resistant (methicillin-resistant S. aureus CIP 106760 and FFHB 29593 (MRSA) and low-level vancomycin-resistant E. faecalis ATCC 51299 (VRE).

Antimicrobial activity

\section{Well diffusion method}

Initially, the microorganisms were cultured on MullerHinton agar for bacteria and Sabouraud agar for yeasts. Then, $100 \mu \mathrm{L}$ of a standardized microorganism suspension, corresponding to $0.5 \mathrm{McF}$ arland, was used to inoculate a Petri dish of solid Mueller-Hinton medium (Sabouraud for yeasts). These suspensions were spread over the medium surface using a sterile swab ${ }^{10-11}$.

Subsequently, agar wells were made of approximately $5.0 \mathrm{~mm}$ in diameter with a Pasteur pipette. Then, $50 \mu \mathrm{L}$ of each sample, negative control (DMSO) and positive controls (vancomycin, amphotericin B or norfloxacin) for Gram positive bacteria (S. aureus, E. faecalis, B. subtilis ; M. smegmatis and S. epidermidis), Gram-negative ( $K$ pneumoniae, $P$. aeruginosa and E. coli) and yeasts $(S$. cerevisiae and $C$. albicans), were added on each of the wells. Plates were incubated at $37{ }^{\circ} \mathrm{C}$ for 24
Os extratos de acetona e de metanol foram preparados usando $10 \mathrm{~g}$ de material vegetal em pó colocado em $200 \mathrm{~mL}$ dos respectivos solventes. Estes extratos foram obtidos utilizando o método de extracção de maceração e ultrassons.

No método de extracção por decocção, os extratos foram preparados por ebulição do material vegetal em água destilada durante 10 minutos. No método de infusão, os extratos foram preparados através da adição de água destilada fervente durante 10 minutos. Os extratos preparados pelo método de extracção de micro-ondas resultaram da junção do material vegetal utilizado com água destilada colocados num aparelho de micro-ondas convencional durante 2 minutos a uma irradiação contínua de $2,45 \mathrm{GHz}$. No método da maceração, o material vegetal e o solvente foram deixados sob agitação à temperatura ambiente. Após 24 horas, o macerado foi filtrado para um balão de fundo redondo e, em seguida, evaporado a pressão reduzida a $40{ }^{\circ} \mathrm{C}$ dando origem a um resíduo. No método de extracção por ultrassons, o matraz com o material vegetal e o solvente foi colocado num aparelho de ultrassons à temperatura ambiente durante 2 horas, após as quais a mistura foi filtrada e solvente evaporado (pressão reduzida e $40{ }^{\circ} \mathrm{C}$ ).

\section{Micro-organismos}

Os micro-organismos utilizados ao longo deste estudo foram sensíveis aos antibióticos (Bacillus subtilis ATCC 6633, Enterococcus faecalis ATCC 29212, Escherichia coli ATCC 25922, Pseudomonas aeruginosa ATCC 27853, Staphylococcus aureus ATCC 25923, Candida albicans ATCC 10231; Mycobacterium smegmatis ATCC 607; Klebsiella pneumoniae ATCC 9997; Saccharomyces cerevivisiae ATCC 9763 e Staphylococcus epidermidis ATCC 12228) e bactérias resistentes (S. aureus resistente à meticilina CIP 106760 e FFHB 29593 (MRSA) e de baixo nível de resistência à vancomicina E. faecalis ATCC 51299 (VRE)).

\section{Actividade Antimicrobiana}

\section{Método de difusão em poços}

Inicialmente, os micro-organismos foram inoculados em meio de Mueller-Hinton para as bactérias e Sabouraud para as leveduras. De seguida, foram utilizados 100 $\mu \mathrm{L}$ de uma suspensão de microorganismo padronizado, correspondente a 0,5 McFarland, para inocular uma placa de Petri com meio de Mueller-Hinton sólido (Sabouraud para as leveduras). Estas suspensões foram espalhadas sobre uma superfície usando uma zaragatoa estéril. ${ }^{[10,11]}$ Posteriormente, os poços foram feitos no agar com aproximadamente 5,0 mm de diâmetro, com uma pipeta de Pasteur. Em seguida, $50 \mu \mathrm{L}$ de cada amostra, do controlo negativo (DMSO) e dos controlos positivos (vancomicina, anfotericina B ou norfloxacina) para as bactérias Gram-positivas ( $S$. aureus, $E$. faecalis, $B$. subtilis, $M$. smegmatis e $S$. epidermidis), Gram-negativa ( $K$. pneumoniae, $P$. aeruginosa e $E$. coli) e leveduras ( $S$. cerevisiae e $C$. albicans), foram adicionados em 
hours. After this period, the diameters of the inhibition zones were measured (no growth) and the results were expressed in millimeters ( $\mathrm{mm}$ ).

The assay was performed under aseptic conditions and in triplicate ${ }^{11}$.

\section{Bioautography Method}

The bioautography is a technique that evaluates qualitatively the antimicrobial activity of mixtures of natural or synthetic substances. This technique is widely used mainly due to the high sensitivity and method ease ${ }^{12}$. This method was only used for the acetonic extracts of $P$. madagascariensis obtained by ultrasound and maceration methods and the acetonic extracts of $P$. neochilus obtained by ultrasound. This selection was based on the activity previously examined in the diffusion method. A Gram-positive reference bacterium, S. aureus, was chosen. The extracts were applied on a TLC plate $(10 \times 10 \mathrm{~cm})$ and then developed using a mixture of $n$ hexane: ethyl acetate $(8: 2 ; \mathrm{v} / \mathrm{v})$. The chromatogram was allowed to dry completely and was placed on a solution of Mueller-Hilton inoculated with $S$. aureus containing tetrazolium chloride $(10 \% \mathrm{w} / \mathrm{v})$, and was incubated overnight at $37^{\circ} \mathrm{C}$.

The development of the plate showed clear zones against a red background indicating inhibition of the bacterial growth by the extract bioactive compounds. The assay was performed four times ${ }^{13}$.

\section{Determination of minimum inhibitory concentration (MIC)}

The assay was performed using the microplate broth microdilution method according to the Clinical and Laboratory Standards Institute (2011). Briefly, $100 \mu \mathrm{L}$ of Mueller-Hilton broth (Sabouraud for yeasts) was placed into each well of a 96 microplate, under aseptic conditions. $100 \mu \mathrm{L}$ of each extract sample, the appropriate positive control of each microorganism and negative controls at a concentration of $1 \mathrm{mg} / \mathrm{mL}$, were added on the first well. Using a multichannel micropipette, a 1:2 microdilution series was made. $10 \mu \mathrm{L}$ of a standardized bacterial suspension, corresponding to 0.5 $\mathrm{McF}$ arland of each microorganism were then placed in all wells. Finally, the plates were incubated at $37{ }^{\circ} \mathrm{C}$ for 24 hours. The microbial growth was evaluated in a microplate absorbance reader (Multiskan FC Thermo Scientific, Loughborough, UK) at $620 \mathrm{~nm}$. The assays were performed in triplicate for all microorganisms ${ }^{3}$. The antimicrobial activity of the extracts was also tested against antibiotic-resistant bacteria $S$. aureus (MRSA - Methicillin-resistant Staphylococcus aureus; FFHB clinical isolate) and E. faecalis (VRE - Vancomycinlow resistant Enterococcus) ${ }^{14}$. cada um dos poços. As placas foram incubadas a $37{ }^{\circ} \mathrm{C}$ durante 24 horas. Após este período, os diâmetros das zonas de inibição foram medidos (ausência de crescimento) e os resultados foram expressos em milímetros ( $\mathrm{mm}$ ). Os ensaios foram realizados sob condições assépticas e em triplicado. ${ }^{[1]}$

\section{Método da Bioautografia}

A Bioautografia é uma técnica que avalia qualitativamente a actividade antimicrobiana de substâncias de origem sintética ou natural contidas numa mistura que são previamente separadas por cromatografia em camada delgada (ccd). Esta técnica é amplamente utilizada devido à sua sensibilidade e facilidade de visualização dos resultados de inibição do crescimento de microrganismos. ${ }^{[12]}$

Este método foi apenas utilizado para os extratos acetónicos do P. madagascariensis obtidos por ultrassons e maceração e para os extratos acetónicos do $P$. neochilus obtidos por ultrassons, que foram escolhidos por apresentarem maior actividade no método da difusão. A bactéria Gram-positiva de referência escolhida para este estudo foi S. aureus.

Os extratos foram aplicados numa placa de ccd $(10 \times 10 \mathrm{~cm})$, esta foi desenvolvida utilizando uma mistura de $n$-hexano: acetato de etilo $(8: 2 ; \mathrm{v} / \mathrm{v})$ e foi deixada a secar completamente. O cromatograma foi colocado numa solução de Mueller-Hilton inoculada com $S$. aureus contendo cloreto de tetrazólio $(10 \% \mathrm{~m} / \mathrm{v})$ e foi incubada durante a noite a $37^{\circ} \mathrm{C}$. Após a incubação a placa apresentou zonas claras contra um fundo vermelho o que indica a inibição do crescimento bacteriano dos compostos bioactivos do extrato. $\mathrm{O}$ ensaio foi realizado quatro vezes. ${ }^{[13]}$

\section{Método da determinação da Concentração Mínima Inibitória (CMI)}

$\mathrm{O}$ ensaio foi realizado pelo método de microdiluição em poços de microplacas de acordo com o Clinical and Laboratory Standards Institute (2011). Resumidamente, $100 \mu \mathrm{L}$ de meio de Mueller-Hilton (Sabouraud para as leveduras) foram colocados em cada poço de uma microplaca de 96 poços, sob condições assépticas. No primeiro poço foram adicionados $100 \mu \mathrm{L}$ de cada amostra de extrato, de igual modo, do apropriado controlo positivo de cada microrganismo e do controlo negativo, a uma concentração de $1 \mathrm{mg} / \mathrm{mL}$. Microdiluições de 1:2 em série foram realizadas, usando uma micropipeta multicanal. Em todos os poços foram colocados $10 \mu \mathrm{L}$ de uma suspensão bacteriana padronizada, correspondente a 0,5 McFarland de cada micro-organismo. Finalmente, as placas foram incubadas a $37^{\circ} \mathrm{C}$ durante 24 horas. $\mathrm{O}$ crescimento microbiano foi avaliado num leitor de microplacas de absorvância (Multiskan FC Thermo Scientific, Loughborough, Reino Unido) a $620 \mathrm{~nm}$. Os ensaios foram realizados em triplicado para todos os micro-organismos. ${ }^{[3]} \mathrm{A}$ atividade antimicrobiana dos extratos também foi testada contra bactérias resistentes a antibióticos S. aureus (MRSA - Staphylococcus aureus resistente à meticilina; FFHB - isolado clínico) e E. faecalis (VRE - Enterococcus de baixa resistência à vancomicina). ${ }^{[14]}$ 


\section{Results}

\section{Well Diffusion method}

The antibacterial activity of the several extracts of the two species of the Plectranthus genus was evaluated by the well diffusion method. Considering all extracts, the acetonic extracts of $P$. madagascariensis obtained by the ultrasound and the maceration methods and the acetonic extract of $P$. neochilus obtained by the ultrasound technique were the more active against the five Gram-positive bacteria tested (5-24 mm of inhibition zone). The highest antibacterial activity was found against bacteria S. aureus (Table 1).

None of the extracts showed significant antibacterial activity against Gram negative bacteria or against the yeasts tested, with inhibition zones identical to that obtained with the negative control DMSO $(5 \mathrm{~mm})$.

It was also noted that none of the extracts showed significant antibacterial activity against Gram-negative bacteria and yeasts.

Table 1 - Antimicrobial activity of the acetonic extracts obtained by the well diffusion method.

\begin{tabular}{|c|c|c|c|c|}
\hline \multirow{2}{*}{\multicolumn{2}{|c|}{ Microrganisms }} & \multicolumn{2}{|c|}{ Plectranthus madagascariensis } & \multirow{3}{*}{$\begin{array}{l}\text { Plectranthus neochilus } \\
\text { Ultrasound } \\
(\mathrm{mm}) \\
8\end{array}$} \\
\hline & & \multirow{2}{*}{$\begin{array}{l}\text { Ultrasound } \\
(\mathrm{mm}) \\
20\end{array}$} & \multirow{2}{*}{$\begin{array}{l}\text { Maceration } \\
(\mathrm{mm}) \\
24\end{array}$} & \\
\hline \multirow{5}{*}{$\begin{array}{l}\text { Gram } \\
\text { Positive } \\
\text { Bacteria }\end{array}$} & S. aureus & & & \\
\hline & E.faecalis & 15 & 5 & 5 \\
\hline & B. subtilis & 24 & 20 & 12 \\
\hline & M. smegmatis & 26 & 26 & 20 \\
\hline & S. epidermidis & 25 & 10 & n.d. \\
\hline \multirow{2}{*}{$\begin{array}{l}\text { Gram } \\
\text { Negative }\end{array}$} & K.pneumoniae & 22 & 25 & 5 \\
\hline & P. aeruginosa & 5 & 5 & 5 \\
\hline \multirow[t]{2}{*}{ Bacteria } & E. coli & 5 & 5 & 5 \\
\hline & C. albicans & 5 & 5 & 5 \\
\hline Yeasts & S. cerevisiae & 5 & 5 & 5 \\
\hline
\end{tabular}

n.d. - not determined 


\section{Resultados}

\section{Método de difusão em poços}

A actividade antimicrobiana dos vários extratos das duas espécies do género Plectranthus foi avaliada pelo método de difusão em poços. De todos os extratos estudados, os extratos acetónicos de P. madagascariensis obtidos por ultrassons e pelo método de maceração e do extrato acetónico do $P$. neochilus obtido pela técnica de ultrassons mostraram atividade contra as cinco bactérias Gram-positivas testadas, com 5-24 mm de zona de inibição. A maior actividade antibacteriana encontrada foi em relação à bactéria $S$. aureus (Tabela 1).

Nenhum dos extratos mostrou actividade antibacteriana significativa contra bactérias Gram negativas nem nas leveduras testadas, apresentando zonas de inibição iguais à obtida com o controlo negativo DMSO (5 mm).

Tabela 1 - Actividade antimicrobiana dos extratos acetónicos pelo método de difusão em poços.

\begin{tabular}{|c|c|c|c|c|}
\hline & & Plectranthu & scariensis & Plectranthus neochilus \\
\hline Micro-orgar & & Ultrassons & Maceração & Ultrassons \\
\hline & S. aureus & 20 & 24 & 8 \\
\hline Bactérias & E. faecalis & 15 & 5 & 5 \\
\hline Gram & B. subtilis & 24 & 20 & 12 \\
\hline Positivo & M. smegmatis & 26 & 26 & 20 \\
\hline & S. epidermidis & 25 & 10 & n.d. \\
\hline Bactérias & K. pneumoniae & 22 & 25 & 5 \\
\hline Gram & P. aeruginosa & 5 & 5 & 5 \\
\hline ivegativo & E. coli & 5 & 5 & 5 \\
\hline & C. albicans & 5 & 5 & 5 \\
\hline & S. cerevisiae & 5 & 5 & 5 \\
\hline
\end{tabular}

n.d. - não determinado 
In this last case, the zones of inhibition were similar to the negative control DMSO $(5 \mathrm{~mm})$.

\section{Bioautography Method}

Concerning the bioautography assays, the three bioactive extracts previously selected (acetonic extracts of $P$. madagascariensis obtained by ultrasound and the maceration method and the acetonic extract of $P$. neochilus obtained by the ultrasound technique) have also shown antibacterial activity against the $S$. aureus bacteria (Figures 1-3). The plates revealed that the antimicrobial compounds were the more polar compounds $(<\mathrm{Rf})$ corresponding to clear zones against a red background, thus indicating inhibition of the bacterial growth.

\section{Método de Bioautografia}

Relativamente aos ensaios de bioautografia, nos três extratos bioactivos selecionados anteriormente (os extratos acetónicos de $P$. madagascariensis obtidos por ultrassons e pelo método de maceração e do extrato acetónico do $P$. neochilus obtido pela técnica de ultrassons) também se observou atividade antibacteriana em relação à bactéria $S$. aureus (Figuras $1 \mathrm{a} 3$ ). Os cromatogramas monstraram que a atividade antimicrobiana corresponde aos compostos mais polares, pois foi na parte inferior do cromatograma $(<\mathrm{Rf})$ que não se observou coloração vermelha característica do crescimento bacteriano.
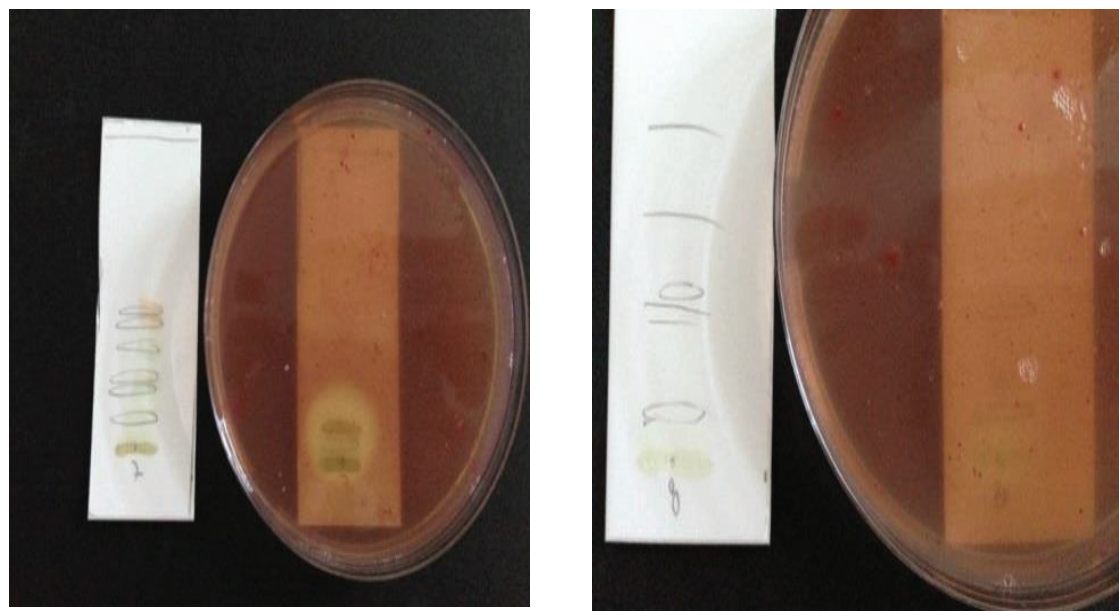

Figure 1/ Figura 1.

Antimicrobial activity observed by the bioautography method of the P. madagascariensis acetonic extract obtained by the ultrasound technique. Atividade antimicrobiana observada pelo método de bioautografia do extrato acetónico de Plectranthus madagascariensis obtido por ultrassons
Figure 2/ Figura 2.

Antimicrobial activity observed by the bioautography method of the P. madagascariensis acetonic extract obtained by the maceration technique. Atividade antimicrobiana obtida pelo método de bioautografia do extrato acetónico do Plectranthus madagascariensis obtido por maceração.

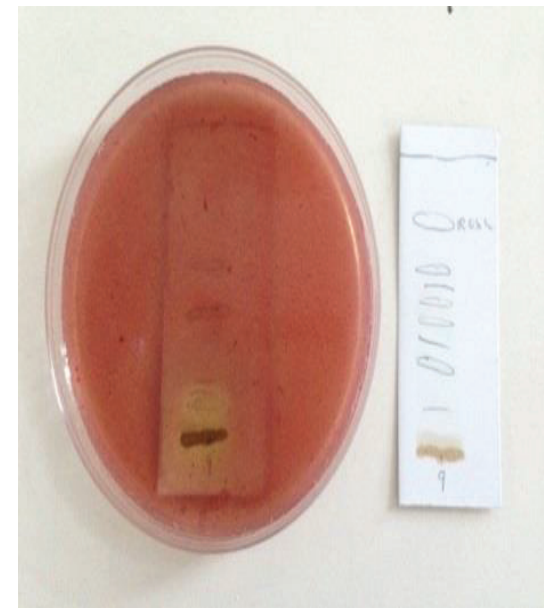

Figure 3/ Figura 3.

Antimicrobial activity observed by the bioautography method of the $P$. neochilus acetonic extract obtained by the ultrasound technique.

Atividade antimicrobiana obtidaatravés do método de bioautografia do extrato acetónico do Plectranthus neochilus obtido por ultrassons. 
Microdilution method: minimum inhibitory concentration evaluation (MIC)

The antimicrobial activity of bioactive extracts previously selected by the well diffusion method and bioautography was further evaluated by the broth microdilution method. The values of minimum inhibitory concentration (MIC) were determined ranging from 250 to $0.49 \mu \mathrm{g} / \mathrm{mL}$ The acetone extract of $P$. madagascariensis obtained by ultrasound method was the most active against all Gram positive bacteria tested (Table 2) (MIC values ranging from 31.25 to $0.49 \mu \mathrm{g} / \mathrm{mL}$ ) and the Gram-negative bacteria $K$. pneumoniae $(<0.49 \mu \mathrm{g} /$ $\mathrm{mL}$ against $<7.81$ for P. neochilus ; Table 2$)$. The acetone extract of $P$. madagascariensis obtained by ultrasound method was also tested against three strains of the antibiotic resistant bacteria MRSA and VRE and the corresponding MIC values were between 31.25 to 0.98 $\mu \mathrm{g} / \mathrm{mL}$ (Table 3).
Método de microdiluição: Determinação da concentração mínima inibitória (CMI)

A atividade antimicrobiana dos extratos bioativos selecionados pelos métodos de difusão em poços e por bioautografia foi ainda avaliada pelo método de microdiluição. Os valores de concentração mínima inibitória (CMI) foram determinados e foram obtidos entre $250 \mathrm{e}$ $0,49 \mu \mathrm{g} / \mathrm{mL}$. O extrato acetónico de $P$. madagascariensis obtido por ultrassons foi o extrato mais ativo contra todas as bactérias de Gram positivo (Tabela 2) testadas (valores de CMI entre 31,25-0,49 $\mu \mathrm{g} / \mathrm{mL}$ ) e a bactéria de Gram negativo K. pneumoniae $(7,81-<0,49 \mu \mathrm{g} / \mathrm{mL}$; Tabela 2). $\mathrm{O}$ extrato acetónico de $P$. madagascariensis obtido por ultrassons foi também testado em três estirpes de bactérias resistentes a antibióticos MRSA e VRE e os correspondentes valores de CMI foram entre 31,25$0,98 \mu \mathrm{g} / \mathrm{mL}$ (Tabela 3).

Table 2/ Tabela 2 - Antimicrobial activity of the acetone extracts by the microdilution method (MIC values ranging between $250-0.49 \mu \mathrm{g} / \mathrm{mL}$ )

Atividade antimicrobiana dos extratos acetónicos pelo método de microdiluição (Valores de MIC entre 250-0,49 $\mu \mathrm{g} / \mathrm{mL}$ )

\begin{tabular}{|c|c|c|c|c|}
\hline \multirow{2}{*}{\multicolumn{2}{|c|}{ Bacteria }} & \multicolumn{2}{|c|}{ P. madagascariensis } & \multirow{2}{*}{$\begin{array}{l}\text { P. neochilus } \\
\text { Ultrasound } \\
\text { Method/ } \\
\text { Ultrassons }\end{array}$} \\
\hline & & $\begin{array}{l}\text { Ultrasound } \\
\text { method/ } \\
\text { Ultrassons }\end{array}$ & $\begin{array}{l}\text { Maceration } \\
\text { Method/ } \\
\text { Maceração }\end{array}$ & \\
\hline \multirow{5}{*}{$\begin{array}{l}\text { Gram positive/ } \\
\text { Gram positivo }\end{array}$} & B.subtilis & 3.91 & 62.5 & 125 \\
\hline & S.aureus & 3.91 & 250 & 250 \\
\hline & S.epidermidis & 7.81 & 62.5 & 62.5 \\
\hline & M.smegmatis & 31.25 & 62.5 & 15.62 \\
\hline & E.faecalis & n.d & 15.62 & n.d \\
\hline $\begin{array}{l}\text { Gram negative/ } \\
\text { Gram negativo }\end{array}$ & K.pneumoniae & $<0.49$ & 3.91 & $<7.81$ \\
\hline
\end{tabular}

n.d. - not determined/ não determinado 
Table 3/ Tabela 3 - Antimicrobial activity of acetonic extract of P. madagascariensis obtained by ultrasound method by the microdilution method against resistant bacteria (MIC values ranging between $250-0.49 \mathrm{ug} / \mathrm{mL}$ ) Atividade antimicrobiana do extrato acetónico de P. madagascariensis obtido por ultrassons pelo método de microdiluição contra bactérias resistentes (valores de CMI entre 250-0,49 $\mu \mathrm{g} / \mathrm{mL}$ ).

\begin{tabular}{|l|c|c|c|}
\hline Bacteria/ & & & \\
Estirpe & S. aureus (MRSA) & S. aureus FFHB & E. faecalis (VRE) \\
\hline MIC & 1.95 & 0.98 & 31.25 \\
\hline
\end{tabular}

\section{Discussion}

In the present study, the antimicrobial activities of two species of the genus Plectranthus (P. neochilus and $P$. madagascarensis) were investigated. Different extraction methods were carried out including infusion, decoction and microwave methods for aqueous extracts and ultrasonic and maceration for acetonic and methanolic extracts. The extraction solvents used were water, acetone and methanol. The objective of this study was to investigate the antimicrobial characteristics of both species and evaluate the bioactivity differences between the different extraction solvents and methods.

In vitro tests for antimicrobial activities were performed using Gram-positive bacteria (S. aureus, E. faecalis, B. subtilis, M.smegmatis and S. epidermidis), Gramnegative bacteria ( $K$. pneumoniae, $P$. aeruginosa and $E$.coli) and yeasts (C. albicans and $S$. cerevisiae). The antimicrobial activity was performed by the well diffusion method to select and identify the bioactive extracts. Only the acetone extracts of P. madagascarensis (obtained by maceration and ultrasound methods) showed inhibition activity against the Gram positive bacteria (Table 1) and showed no significant activity against the Gram negative bacteria and yeasts. This is in agreement with other studies previously published ${ }^{7}$. Aqueous and methanolic extracts were shown to be inactive against all the microorganisms tested.

The previously selected active extracts were evaluated by the broth microdilution method to determine the MIC values. The antimicrobial activity against three antibiotic-resistant bacteria, MRSA and VRE, was also evaluated by the same method in the most active acetonic extract previously selected ( $P$. madagascarensis obtained by ultrasound methods). Through the bioautography method it was observed that the most polar compounds are responsible for the antimicrobial activity. Other studies in the genus Plectranthus species $^{2,15,16}$

\section{Discussão}

No presente estudo foram pesquisadas as atividades antimicrobianas de duas espécies do género Plectranthus (P. neochilus e P. madagascarensis). Foram realizados diferentes métodos de extração tendo sido utilizado os métodos de infusão, decocção e microondas para os extratos aquosos e o método de ultrassons e maceração para os extratos acetónicos e metanólicos, utilizando como solventes de extração a água, a acetona e o metanol. O objetivo deste estudo foi pesquisar as características antimicrobianas das duas espécies e observar as diferenças na bioatividade entre os diferentes solventes e métodos de extração.

Os ensaios in vitro da atividade antimicrobiana foram realizados utilizando bactérias de Gram positivo ( $S$. aureus, E. faecalis, B. subtilis, $M$. smegmatis e $S$. epidermidis), bactérias de Gram negativo ( $K$. pneumoniae, $P$. aeruginosa e E. coli) e leveduras (C. albicans e S. cerevisiae). A atividade antimicrobiana foi avaliada pelo método de difusão em poços, de modo a selecionar e identificar os extratos bioativos. Apenas os extratos acetónicos de P. madagascarensis (obtidos por maceração e ultrassons) demostraram resultados positivos em relação às bactérias Gram positivo (Tabela 1) mas não apresentaram atividade significativa contra as bactérias de Gram negativas e leveduras, o que está de acordo com outros estudos publicados anteriormente. ${ }^{[7]}$ Os extratos aquosos e metanólicos mostraram ser inativos em todos os micro-organismos testados.

Os extratos que demostraram ser ativos foram avaliados pelo método de microdiluição de modo a determinar os valores de CMI. A atividade antimicrobiana contra três bactérias resistentes a antibióticos MRSA e VRE foi também avaliada pelo mesmo método, no extrato mais ativo seleccionado anteriormente (P. madagascarensis obtido por ultrassons). Através do método de bioautografia foi observado que os compostos mais polares são 
describe compounds such as abietane-royleanone type, phenolic compounds (such as rosmarinic acid), essential oils and flavonoids as responsible for the antimicrobial activity. The cytotoxicity of the extracts that have shown antimicrobial activity should be evaluated in order to verify that the inhibition of microbial growth is due to its antimicrobial activity and not to its constituents toxicity. Future phytochemical studies must be performed to reveal the structural identification (by spectroscopic methods) of the compounds responsible for the antimicrobial activity. Thus, from this study Plectranthus genus and in particular the species P. madagascariensis and $P$. neochilus demonstrate to be important sources of antimicrobial compounds with potential for developing new antibiotics.

\section{Conclusion}

In this study, only acetonic extracts of the $P$. madagascariensis and $P$. neochilus showed activity against Gram-positive bacteria. The aqueous and methanolic extracts were inactive.

In the study, we may conclude that extracts of $P$. madagascariensis and P.neochilus may be considered a potential source of new bioactive chemicals and thus promising drug candidates for therapeutic critical areas such as infectious diseases.

\section{Conflict of interests}

The author declares that there are no financial or personal relationships that could be viewed as potential conflict of interest. os responsáveis pela atividade antimicrobiana. Outros estudos em espécies do género Plectranthus ${ }^{[2][15][16]}$ citam os compostos do tipo abietano-roileanona, compostos fenólicos (como o ácido rosmarínico), óleos essenciais e flavonóides como os responsáveis pela atividade antimicrobiana.

A citotoxicidade dos extratos que demostraram atividade antimicrobiana deverá ser avaliada de modo a verificar que a inibição do crescimento microbiano se deve à sua atividade antimicrobiana e não à toxicidade dos seus constituintes.

Outros estudos fitoquímicos deverão ser realizados, no futuro, para identificar (através de métodos espectroscópicos) os compostos bioativos responsáveis pela atividade antimicrobiana observada. Do presente estudo concluiu-se que o género Plectranthus nomeadamente as espécies $P$. madagascariensis e $P$. neochilus demonstram ser fontes importantes de compostos antimicrobianos com potencial para o desenvolvimento de novos antibióticos.

\section{Conclusão}

No presente estudo, foram preparados extratos aquosos, acetónicos e metanólicos de $P$. madagascariensis e $P$. neochilus utilizando vários métodos de extração (infusão, decocção, maceração, extração assistida por micro-ondas e por ultrassom e com fluidos no estado supercrítico). Todos os extratos foram testados contra bactérias de Gram-positivo, de Gram-negativo e leveduras, mas apenas os extratos acetónicos de $P$. madagascariensis (obtidos por maceração e ultrassons) e $P$. neochilus (obtidos por ultrassons) mostraram atividade em relação às bactérias de Gram positivo testadas. Com este estudo é possível concluir que os extratos de $P$. madagascariensis e $P$. neochilus poderão ser uma potencial fonte de novas substâncias bioativas importantes sendo, por conseguinte, candidatos promissores a fármacos em áreas terapêuticas fundamentais como as doenças infecciosas.

\section{Conflitos de Interesse}

O autor declara que não existem relações financeiras ou pessoais que pudessem ser vistas como potenciais conflitos de interesse. 


\section{References/ Referências}

[1] D. Guimarães, Luciano da Silva Momesso, Mônica Tallarico Pupo; Antibióticos: Importância terapêutica e perspectivas para a descoberta, 2010, Quim. Nova, 33, 3, 667679.

[2] P.Rijo C.Faustino and M. Fátima Simões, Antimicrobial natural products from Plectranthus plants, Microbial pathogens and strategies for combating them: science, technology and education (A. Méndez-Vilas, Ed.), 2013.

[3] P.Rijo, Marina Batista, Marisa Matos, Helga Rocha, Sandra Jesus, M. Fátima Simões, Screening of antioxidant and antimicrobial activities on Plectranthus spp. Extacts; Biomedical and biopharmaceutical research, 2012, 9, 2: 225-235.

[4] Renata Kubínova, Radka Pořízková, Alice Navrátilová, Oldřich Farsa, Zuzana Hanáková, Adriana Bačinská, Alois Č́žek, and Marie Valentová, Antimicrobial and enzyme inhibitory activities of the constituents of

Plectranthus madagascariensis (Pers.) Benth, 2014, J Enzyme Inhib Med Chem., 29, 5, 749-52.

[5] Nahed M. Waly and and Sabah H. El Gayed, Botanical and biological studies of Plectranthus tenuiflorus (Vatke) Agnew. (Lamiaceae) growing in Saudi Arabia, , International Journal of Life Sciences \& Pharma Research, 2012, 2, 2. .

[6] Catherine W. Lukhobe, Simmonds MS, Paton AJ., Plectranthus: A review of ethnobotanical uses, Journal of Ethnopharmacology, 2006, 103 ()1-24.

[7] Gaspar-Marques, Patrícia Rijo, M. Fátima Simões, M. Aida Duarte e Benjamin Rodriguez, Abietanes from Plectranthus grandidentatus and P.hereroensis against methicillin- and vancomycinresistant bacteria, Phytomedicine, 2006, 13, 267-271.
[8] P.Rijo, Phytochemical study and biological activities of diterpenes and derivatives from Plectranthus species, $\mathrm{PhD}$ thesis of University of Lisbon, Lisbon, Portugal, 2011.

[9] Tagreed Alsufyani Asif fatani, Faten Khorshedm, Soad Shaker and Hassan A. H. Albar 1, phytochemical composition and antimicrobial activities of the essential oil from plectranthus tenuiflourus growing in Saudi Arabia.

[10] E.Alves, et al, Estudo comparativo de técnicas de screening para avaliação da actividade antibacteriana de extratos brutos de espécies vegetais e de substâncias puras, Quim. Nova, 200831,5, 1224-1229.

[11] P.Rijo, Diogo Matias, Ana S. Fernandes, M. Fátima Simões, Marisa Nicolai and Catarina Pinto Reis, , Antimicrobial Plant Extracts Encapsulated into Polymeric Beads for Potential Application on the Skin; Polymers 2014, 6, 479-490.

[12] L.McGaw Lyndy Joy McGaw, Victor Patrick Bagla, Paul Anton Steenkamp, Gerda Fouche, Jana Olivier, Jacobus Nicolaas Eloff and Martin Steven Myer, Antifungal and antibacterial activity and chemicalcomposition of polar and non-polar extracts of Athrixia phylicoides determined using bioautography and HPLC, BMC Complementary and Alternative Medicine 2013.
[13] M.Bastos, Maria Lysete A Bastos, Maria Raquel F Lima, Lucia M Conserva, Vânia S Andrade, Eliana MM Rocha and Rosangela PL Lemos ,, Studies on the antimicrobial activity and brine shrimp toxicity of Zeyheria tuberculosa extracts and their main constituints, Annals of Clinical Microbiology and Antimicrobials 2009, 8:16.

[14] P.Rijo, Duarte A, Francisco AP, SemedoLemsaddek T, Simões MF., , In vitro antimicrobial activity of royleanone derivates against Gram positive bacterial pathogens,Phytother Res. 2014, 28, 1, 76-81.

[15] M.Mogib, M. Abdel-Mogib, H. A. Albar and S. M. Batterjee, , review: Chemistry of the genus Plectranthus, Molecules 2002, 7, 2, 271-301

[16] Renée J. Grayer, Maria R. Eckert, Andrew Lever, Nigel C. Veitch, Geoffrey C. Kite, Alan J. Paton, . Distribution of exudate flavonoids in the genus Plectranthus, Biochemical Systematics and Ecology, 2010, 38, 3, 335341 . 